\title{
Inhibition of lymphangiogenesis in vitro and in vivo by the multikinase inhibitor nintedanib
}

This article was published in the following Dove Press journal:

Drug Design, Development and Therapy

5 April 2017

Number of times this article has been viewed

\section{Tong Lin ${ }^{1,2}$ \\ Lan Gong ${ }^{1,2}$}

'Department of Ophthalmology, Eye and ENT Hospital, Fudan University,

${ }^{2}$ Key Laboratory of Myopia, Ministry of Health, Shanghai, People's Republic of China
Correspondence: Lan Gong Department of Ophthalmology, Eye and ENT Hospital, Fudan University, 83 Fenyang Road, Shanghai 20003I, People's Republic of China Tel/fax +86 2l 643I 0068 Email13501798683@I39.com
Purpose: To investigate the feasibility of nintedanib, a novel triple angiokinase inhibitor, for inhibiting lymphatic endothelial cell (LEC)-induced lymphangiogenesis in vitro and inflammatory corneal lymphangiogenesis in vivo.

Materials and methods: Methylthiazolyldiphenyl-tetrazolium bromide (MTT) test, transwell system, and tube-formation assay were used to evaluate the effects of nintedanib on the proliferation, migration, and tube formation of LECs stimulated by vascular endothelial growth factor-C (VEGF-C), basic fibroblast growth factor (bFGF), or platelet-derived growth factor-BB (PDGF-BB). The murine model of suture-induced corneal neovascularization was used to assess the anti-hemangiogenic and anti-lymphangiogenic effects of nintedanib via systemic and topical applications. Corneal flatmounts were stained with lymphatic vessel endothelial hyaluronan receptor-1 (LYVE-1) and CD31, and the areas of involved blood and lymph vessels were analyzed morphometrically. Corneal cryosections were stained with F4/80 to evaluate inflammatory cell recruitment.

Results: We observed a significant enhanced effect of LEC proliferation, migration, and tube formation with the administration of VEGF-C, PDGF-BB, and bFGF, respectively, which was diminished by nintedanib. Both topical and systemic applications of nintedanib inhibited suture-induced hemangiogenesis and lymphangiogenesis in the murine cornea. A reduction in $\mathrm{F} 4 / 80^{+}$cell infiltration was observed at day 14 after corneal suture for both systemic and topical applications of nintedanib. In comparison with controls, $61 \%$ of $\mathrm{F} 4 / 80^{+}$cell recruitment was inhibited via the systemic application of nintedanib, while $49 \%$ of $\mathrm{F} 4 / 80^{+}$cell recruitment was inhibited with the topical application of nintedanib.

Conclusion: Nintedanib was shown to inhibit in vitro lymphangiogenesis stimulated by VEGF-C, bFGF, and PDGF-BB. Applied topically or systemically, it effectively inhibited corneal hemangiogenesis and lymphangiogenesis, accompanied by reduced inflammatory cell recruitment, which represents a new promising treatment for graft rejection after penetrating keratoplasty.

Keywords: kinase inhibitor, lymphangiogenesis, in vitro, in vivo

\section{Introduction}

Lymphangiogenesis is defined as the formation of new lymphatic vessels from preexisting ones, and it involves proliferation, migration, and tube formation of the lymphatic endothelial cells (LECs). ${ }^{1}$ Growth factors, adhesion molecules, chemokines, and the extracellular matrix play important roles in lymphangiogenesis. ${ }^{2-4}$ Previous studies have shown that an extensive variety of proangiogenic factors, including vascular endothelial growth factor (VEGF), fibroblast growth factor (FGF), and platelet-derived growth factor (PDGF), could be also involved in the development of lymphangiogenesis. ${ }^{5-8}$ It is well established that vascular endothelial growth factor 
(VEGF) receptor-3 (VEGFR-3) is a specific receptor of VEGF-C and VEGF-D, and upregulation of the expression of VEGF-C and VEGF-D promotes lymphangiogenesis. ${ }^{9,10}$ Meanwhile, basic fibroblast growth factor (bFGF) has been demonstrated to promote lymphangiogenesis directly and to act in an indirect role involving the upregulation of VEGF-C and VEGF-D expression in lymphangiogenesis. ${ }^{11}$ It has previously been reported that PDGF-BB can also stimulate the proliferation and migration of LECs, and blockage of the VEGF-C/VEGF-D/VEGFR-3 pathway fails to inhibit PDGF-BB-induced lymphangiogenesis; this suggests that PDGF-BB also has a direct effect on LECs. ${ }^{12}$

In pathological conditions, such as transplant rejection and cancer metastasis, numerous angiogenic factors arise to stimulate angiogenesis and lymphangiogenesis; this could contribute to the exacerbation of these diseases. ${ }^{13-17}$ Inhibition of lymphangiogenesis is suggested to be a powerful selective therapy for reducing lymphatic metastasis of tumor cells and graft rejection. Recently, a novel, potent, triple angiokinase inhibitor called nintedanib was developed. Nintedanib competitively binds to the adenosine5 'triphosphate (ATP)-binding site of receptor tyrosine kinases (RTKs), including VEGFR, fibroblast growth factor receptor (FGFR), and platelet-derived growth factor receptor (PDGFR) and then inhibits their downstream intracellular signaling. ${ }^{18}$ Nintedanib has displayed potent antiangiogenic effects in vitro by inhibiting endothelial, pericyte, and smooth muscle cell proliferation in culture and antitumor activity in several tumor xenograft models in vivo by reducing tumor microvascular density and the number of perivascular cells. ${ }^{18,19}$ Moreover, this multi-targeted angiokinase inhibitor has also demonstrated to be effective in several human cancer trials. ${ }^{20-22}$ Furthermore, nintedanib was found to be an effective and potent inhibitor of the pre-retinal pathological neovascularization in a murine model of vasoproliferative retinopathy. ${ }^{23}$ Although the antiangiogenic effect of nintedanib is well recognized, its effect on lymphangiogenesis remains unclear. This investigation was designed to determine the feasibility of the multikinase inhibitor, nintedanib, for inhibiting LEC-induced lymphangiogenesis in vitro and inflammatory corneal lymphangiogenesis in vivo.

\section{Materials and methods}

\section{Mice}

Six-week-old male C57BL/6 mice purchased from SIPPR-Bk Laboratory Animal Co., Ltd. (Shanghai, China) were used throughout this study. The animals were maintained with a 12-h light-dark cycle and had free access to food and water.
Animal experiments were performed in accordance with the Association for Research in Vision and Ophthalmology (ARVO) Statement for the Use of Animals in Ophthalmic and Vision Research; the study was approved by Fudan University's Animal Ethical Committee.

\section{Cell culture}

Primary human lymphatic endothelial cells (HLECs) were purchased from PromoCell (Heidelberg, Germany) and cultured in endothelial basal medium (EBM; Lonza, Walkersville, MD, USA) supplemented with 10\% fetal calf serum (FCS; Biowest, Riverside, MO, USA) and other supplements, as previously described. ${ }^{24}$ HLECs at passages 4-8 were used in this study.

\section{Nintedanib}

Nintedanib was purchased from Selleckchem (Houston, TX, USA). For in vitro assays, nintedanib was solubilized in dimethyl sulfoxide (DMSO; Sigma-Aldrich, St Louis, MO, USA) and used at $0.01,0.1$, or $1 \mu \mathrm{M}$ in culture medium. For in vivo assays, nintedanib was solubilized in $0.5 \%$ carboxymethyl cellulose. Fifty $\mathrm{mg} / \mathrm{kg}$ was administered daily by oral gavage, and $5 \mathrm{mg} / \mathrm{mL}$ was used as topical administration. Control mice received vehicle.

\section{Cell proliferation assay}

The HLEC proliferation assay was performed using methylthiazolyldiphenyl-tetrazolium bromide (MTT; Sigma-Aldrich) assay. HLECs were seeded onto each well of 96-well plates in a total volume of $150 \mu \mathrm{L}$ EBM containing $2 \%$ ( $\mathrm{vol} / \mathrm{vol}) \mathrm{FCS}$ at a density of $5 \times 10^{3}$ cells/ well. After incubation for $24 \mathrm{~h}$, cells were treated with 50 ng/mL recombinant VEGF-C (PeproTech, Rocky Hill, NJ, USA), PDGF-BB (PeproTech), or bFGF (PeproTech) in the presence or absence of nintedanib at concentrations of $0.01,0.1$, and $1 \mu \mathrm{M}$. The control cells were treated with $0.1 \%$ DMSO. After incubation at $37^{\circ} \mathrm{C}$ for $48 \mathrm{~h}, 20 \mu \mathrm{L}$ of MTT solution ( $5 \mathrm{mg} / \mathrm{mL}$ ) was added to each well, and the cells continued to be incubated for $4 \mathrm{~h}$. Following removal of the medium containing MTT, $200 \mu \mathrm{L}$ of DMSO was added to dissolve the formazan crystals formed by live cells. The absorbance was measured at $490 \mathrm{~nm}$ with an absorbance microplate reader (BioTek, Winooski, VT, USA). The assay was repeated six times.

\section{Migration assay}

A transwell system (Corning Costar, Acton, MA, USA) was used to study HLECs' ability to migrate through a micropore 
nitrocellulose filter (8- $\mu \mathrm{m}$ pore size). In brief, each stimulating factor (VEGF-C, PDGF-BB, or bFGF) was added to the lower chamber at a concentration of $50 \mathrm{ng} / \mathrm{mL}$ in $2 \%$ ( $\mathrm{vol} / \mathrm{vol}$ ) FCS-basal EBM. Approximately $1 \times 10^{5}$ HLECs in serum-free $\mathrm{EBM}$ in the presence or absence of $0.1 \mu \mathrm{M}$ nintedanib were seeded in each well of the upper chamber. The control cells were treated with $0.1 \%$ DMSO. The cells were incubated for $12 \mathrm{~h}$. Non-migrating cells were removed from the top surface of the membrane using a cotton swab. Migrating cells adhering to the undersurface of the filters were fixed with methanol, followed by staining with a 4',6-diamidino-2phenylindole (DAPI, 1:1,000; Sigma-Aldrich) solution, and observed using a fluorescence microscope (DM 4000B; Leica Microsystems, Wetzlar, Germany), as described in a previous study. ${ }^{25}$ The cells that migrated into the lower chamber were counted in five fields for each well. The experiments were performed in triplicate.

\section{Tube-formation assay}

Tube formation was assayed as previously described. ${ }^{26}$ In brief, $200 \mu \mathrm{L}$ of Matrigel (BD Biosciences, Bedford, MA, USA) was added to each well in 24-well plates and allowed to polymerize for $30 \mathrm{~min}$ at $37^{\circ} \mathrm{C}$. After trypsinization, HLECs were seeded on the gel at a density of $1 \times 10^{5}$ cells/well and incubated in the medium containing VEGF-C, FGF-2, or PDGF-BB in the presence or absence of $0.1 \mu \mathrm{M}$ nintedanib for $24 \mathrm{~h}$. The control cells were treated with $0.1 \%$ DMSO. Capillary-like structures organized by the cells were viewed and photographed at a magnification of $200 \times$. The area and length of the tube network in five fields for each well were quantified by ImageJ software (National Institutes of Health). The experiment was performed in triplicate.

\section{Corneal neovascularization assay immunostainings}

The mouse model of suture-induced inflammatory corneal neovascularization was used as previously described. ${ }^{27}$ First, each animal was deeply anesthetized with an intramuscular injection of ketamine $(8 \mathrm{mg} / \mathrm{kg})$ and xylazine $(0.1 \mathrm{~mL} / \mathrm{kg})$. The central cornea was marked with a $2 \mathrm{~mm}$ diameter trephine, and three 11-0 nylon sutures (Lingqiao; Ningbo Medical Needle Co., Ltd., Ningbo, China) were then placed in the intrastromal position extending over $120^{\circ}$ of the corneal circumference to obtain standardized angiogenic responses. Sutures were left in place for 2 weeks.

The systemic treatment group received nintedanib (50 mg/kg, daily) by oral gavage for 14 days after suture surgery. Control mice received equal amounts of the substance vehicle. For enhancing the penetration of nintedanib into the corneal stroma, the central $2 \mathrm{~mm}$ of the corneal epithelium was scraped off before suturing, and the mice received nintedanib ( $5 \mathrm{mg} / \mathrm{mL}$, four times daily) in eye drops for 12 days. Control mice received an equal volume of the substance vehicle. After 2 weeks, mice were sacrificed and corneas were prepared for immunofluorescence assay.

\section{Corneal immunostainings}

Corneal whole mounts were used to determine corneal lymphangiogenesis and hemangiogenesis. Mice were sacrificed at planned times, and the eyes were removed and dissected; following this, whole-mounted corneas were fixed in $4 \%$ paraformaldehyde overnight at $4^{\circ} \mathrm{C}$ and blocked in 5\% donkey serum albumin (Solarbio, Beijing, China) for $1 \mathrm{~h}$. For double lymphatic vessel endothelial hyaluronan receptor-1 (LYVE-1) and CD31 immunostainings, corneas were incubated overnight with polyclonal rabbit anti-mouse LYVE-1 (1/200; Abcam, Cambridge, UK) and rat anti-mouse CD31 (1/100; BD Biosciences Pharmingen, San Jose, CA, USA) antibodies, as described previously. ${ }^{28}$ Following this, incubations with Alexa Fluor 488-coupled donkey anti-rabbit antibody (1/200; Abcam) and Cy3-conjugated donkey anti-rat immunoglobulin $\mathrm{G}$ (IgG; Jackson ImmunoResearch, West Grove, PA, USA) were performed overnight. Corneas flat mounted on a microscope slide with antifade solution (AR1109; Boster, Wuhan, China) were examined using a confocal microscope (TCS SP8; Leica, Heidelberg, Germany). Pictures were taken at $100 \times$ magnification and automatically assembled to reconstitute the whole cornea picture. The area of corneal lymphangiogenesis and hemangiogenesis was quantified by Image J software.

For corneal inflammatory cell recruitment assay, corneal cryosections $(8 \mu \mathrm{m})$ were fixed in $4 \%$ paraformaldehyde for 5 min. ${ }^{29}$ After blocking with 5\% donkey serum albumin in phosphate-buffered saline (PBS), sections were incubated overnight at $4{ }^{\circ} \mathrm{C}$ with rabbit polyclonal $\mathrm{F} 4 / 80$ antibody (1:100; Abcam). After washing with PBS, the sections were incubated for $2 \mathrm{~h}$ at room temperature with Alexa Fluor 488-coupled donkey anti-rabbit antibody (1/200). Next, sections were washed with PBS, followed by staining with a DAPI $(1: 1,000)$ solution; pictures were taken at $400 \times$ magnification using a fluorescence microscope (DM 4000B). Five representative pictures per cornea were taken, and the $\mathrm{F} 4 / 80^{+}$cells were counted in each section. The 
mean cell number of the control section was set as $100 \%$; the numbers of $\mathrm{F} 4 / 80^{+}$cells per section were then related to this value (cell ratio).

\section{Statistical analysis}

Data are expressed as the mean \pm standard deviation (SD). The Student's $t$-test and one-way analysis of variance with Scheffe's post hoc multiple comparison analysis were performed to analyze the data statistically. A value of $P<0.05$ was considered as statistically significant.

\section{Results}

\section{In vitro effect of nintedanib on lymphangiogenesis}

Effect of nintedanib on growth factor-induced proliferation of HLECs

We observed a significant pro-proliferative effect of VEGF-C, PDGF-BB, and bFGF on HLECs compared with the negative control group (all $P<0.01$; Figure $1 \mathrm{~A}-\mathrm{C}$ ). However, the enhanced effect was significantly eliminated when nintedanib was administered at a concentration of $\geq 0.1 \mu \mathrm{M}$, and the inhibiting effect exhibited concentration dependence (Figure $1 \mathrm{~A}-\mathrm{C}$ ).

\section{Nintedanib suppresses growth factor-induced migration of HLECs}

Next, we investigated the effect of nintedanib on growth factor-induced migration of HLECs at a concentration of $0.1 \mu \mathrm{M}$. As expected, VEGF-C, PDGF-BB, and bFGF $(50 \mathrm{ng} / \mathrm{mL})$ significantly stimulated migration of HLECs from the upper chamber to the bottom of the filter (Figure 2). The migration of HLECs stimulated by each growth factor was suppressed with the administration of $0.1 \mu \mathrm{M}$ nintedanib (all $P<0.05$; Figure 2E).

\section{Nintedanib inhibits growth factor-induced tube} formation of HLECs

Meanwhile, we investigated the effect of nintedanib on growth factor-induced tube formation of HLECs at a concentration of $0.1 \mu \mathrm{M}$. After incubation for $24 \mathrm{~h}$, the cells organized into capillary-like structures in Matrigel basement membrane matrix. Tube formation of HLECs was evaluated in terms
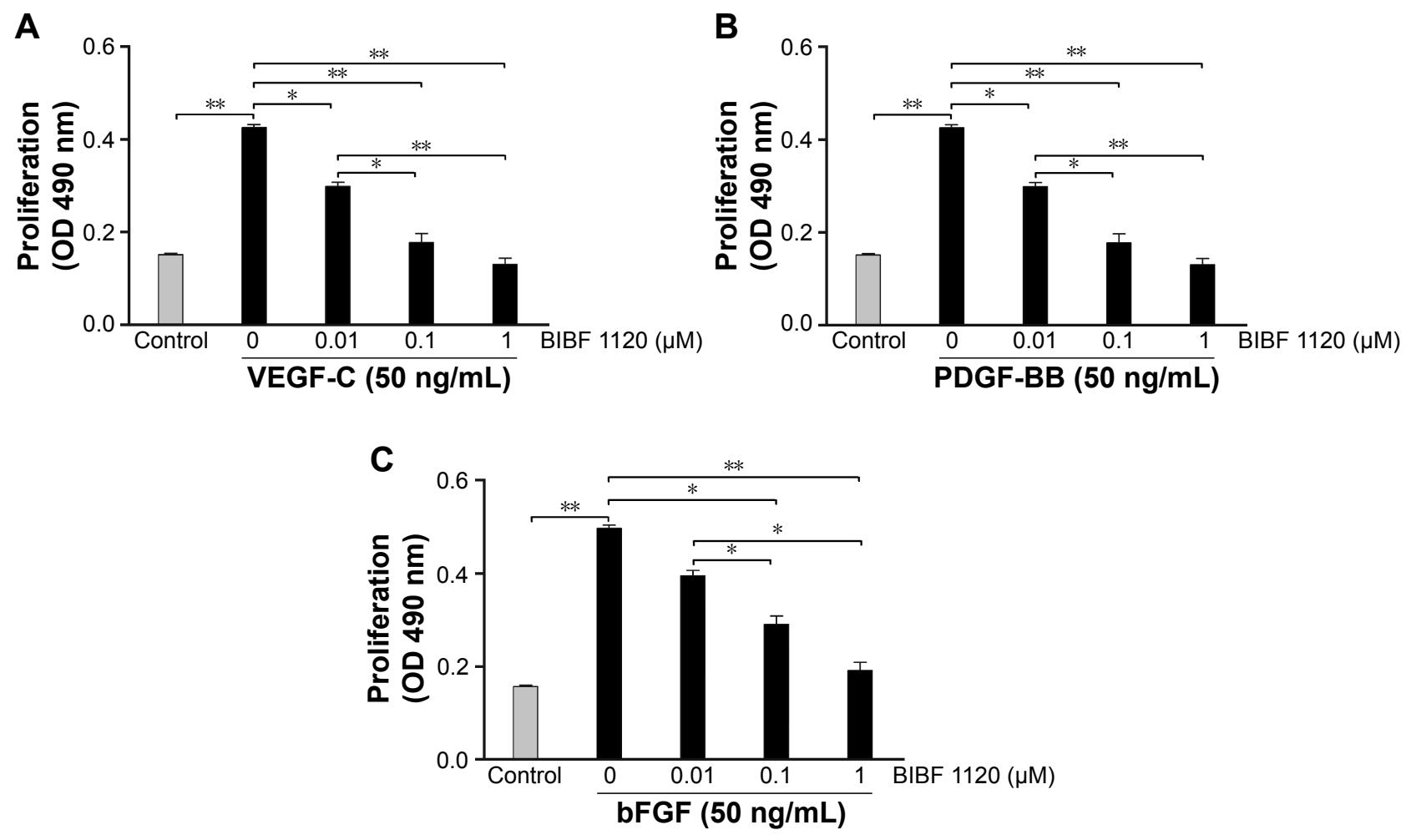

Figure I Effect of BIBF II 20 (nintedanib) on growth factor-induced proliferation of HLECs.

Notes: (A) VEGF-C $(50 \mathrm{ng} / \mathrm{mL})$ enhanced proliferation of HLECs significantly. The enhanced effect was significantly reduced by $0.01 \mu M$ nintedanib $(P<0.05)$ and almost eliminated by $0.1 \mu \mathrm{M}$ nintedanib $(P<0.0 \mathrm{I})$. (B) PDGF-BB $(50 \mathrm{ng} / \mathrm{mL})$ enhanced proliferation of HLECs significantly. The enhanced effect was significantly reduced by $0.0 \mathrm{I} \mu \mathrm{M}$ nintedanib $(P<0.05)$ and almost eliminated by $0.1 \mu \mathrm{M}$ nintedanib $(P<0.0 \mathrm{I})$. (C) A concentration of $50 \mathrm{ng} / \mathrm{mL}$ of bFGF enhanced proliferation of $\mathrm{HLECs}$ significantly. The enhanced effect was significantly reduced by $0.1 \mu \mathrm{M}$ nintedanib $(P<0.05)$ and almost eliminated by I $\mu \mathrm{M}$ nintedanib $(P<0.0 \mathrm{I})$. $* P$-value $<0.05$. $* * P$-value $<0.0 \mathrm{I}$.

Abbreviations: HLECs, human lymphatic endothelial cells; VEGF-C, vascular endothelial growth factor-C; PDGF-BB, platelet-derived growth factor-BB; bFGF, basic fibroblast growth factor; OD, optical density. 

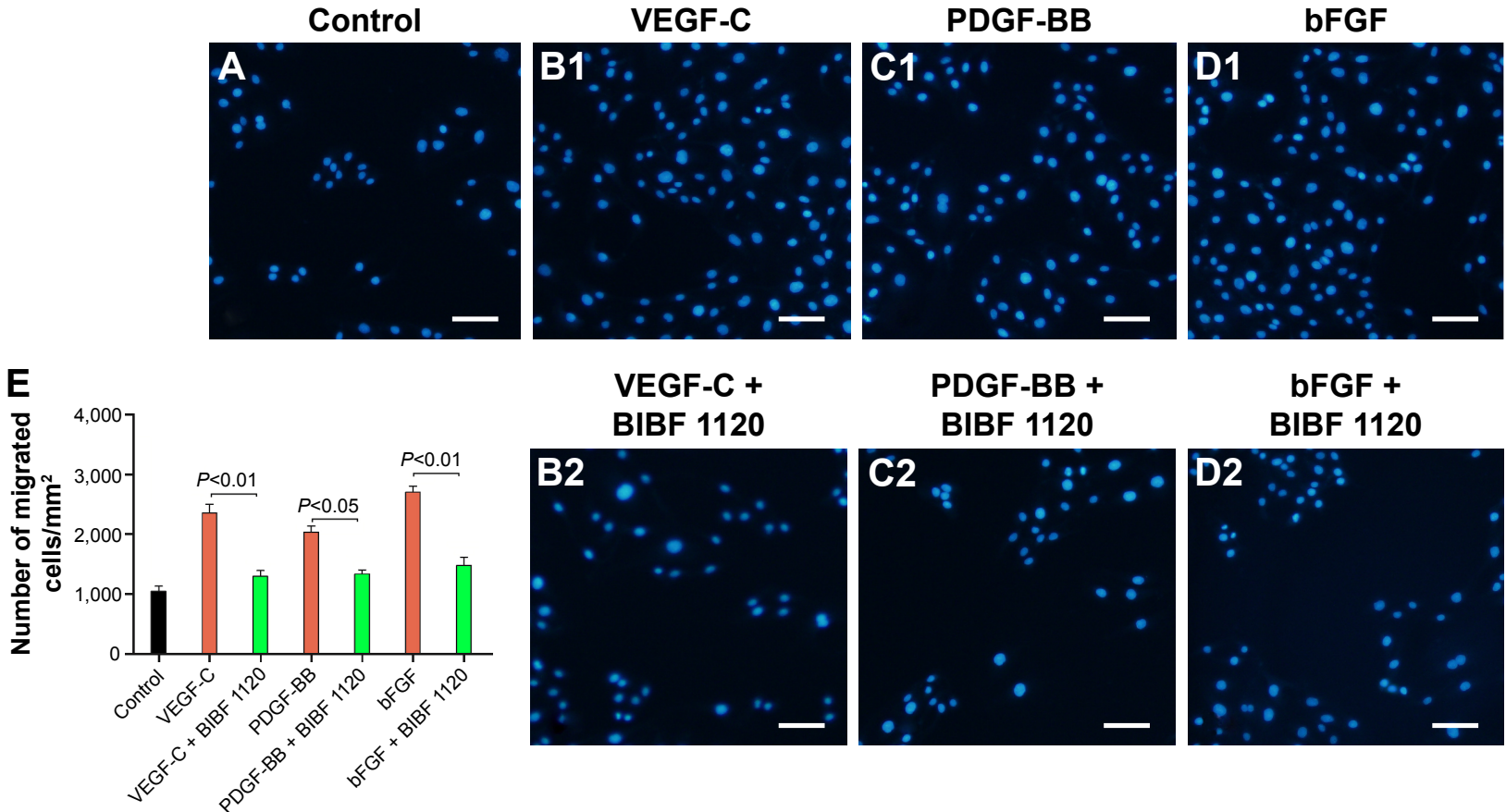

VEGF-C + BIBF 1120

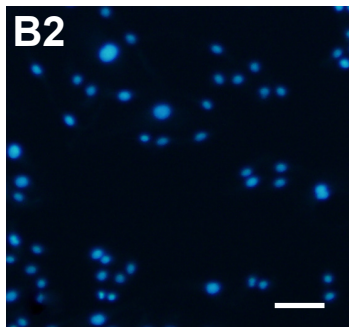

PDGF-BB + BIBF 1120

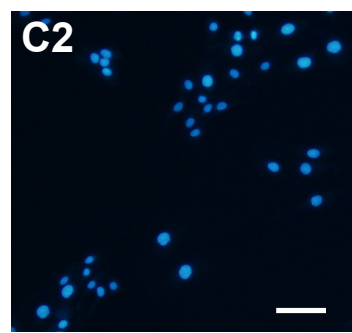

bFGF + BIBF 1120

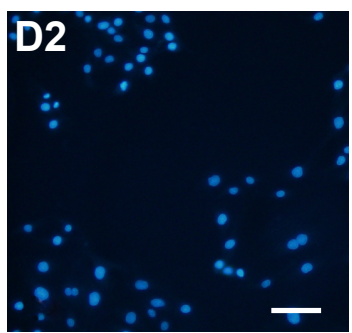

Figure 2 BIBF II 20 (nintedanib) suppressed growth factor-induced migration of HLECs.

Notes: (A) Cells treated with $0.1 \%$ DMSO were used as controls. (B I and B2) VEGF-C $(50 \mathrm{ng} / \mathrm{mL})$ promoted migration of HLECs significantly, and the enhanced effect was significantly reduced by $0.1 \mu \mathrm{M}$ nintedanib. (CI and $\mathbf{C 2})$ PDGF-BB $(50 \mathrm{ng} / \mathrm{mL})$ promoted migration of HLECs significantly, and the enhanced effect was significantly reduced by $0.1 \mu \mathrm{M}$ nintedanib. (DI and D2) A concentration of $50 \mathrm{ng} / \mathrm{mL}$ of bFGF enhanced migration of HLECs significantly, and the enhanced effect was significantly reduced by $0.1 \mu \mathrm{M}$ nintedanib. (E) The number of migrated cells stimulated by VEGF-C, PDGF-BB, or bFGF at a concentration of $50 \mathrm{ng} / \mathrm{mL}$ in the presence or absence of $0.1 \mu M$ nintedanib. A-D at 200× magnification; scale bar $=50 \mu \mathrm{m}$.

Abbreviations: HLECs, human lymphatic endothelial cells; DMSO, dimethyl sulfoxide; VEGF-C, vascular endothelial growth factor-C; PDGF-BB, platelet-derived growth factor-BB; bFGF, basic fibroblast growth factor.

of the length and area of the tube structures. Both the length and area of the tube structures clearly increased with the administration of VEGF-C, PDGF-BB, or bFGF at a concentration of $50 \mathrm{ng} / \mathrm{mL}$ (Figure 3). The tube formation of HLECs stimulated by each growth factor was significantly suppressed by $0.1 \mu \mathrm{M}$ nintedanib (all $P<0.05$; Figure $3 \mathrm{E}$ and $\mathrm{F}$ ).

\section{In vivo effect of nintedanib on lymphangiogenesis}

Effect of systemic application of nintedanib on hemangiogenesis and lymphangiogenesis

We investigated the effect of systemic application of nintedanib on the outgrowth of blood and lymphatic vessels in a suture-induced corneal neovascularization assay. The treatment group $(\mathrm{n}=8)$ showed a significant decrease in angiogenesis $(P<0.01)$ and lymphangiogenesis $(P<0.01)$ compared with the controls ( $n=8$; Figure 4$)$.

\section{Effect of topical application of nintedanib on hemangiogenesis and lymphangiogenesis}

We sought to determine whether the topical application of nintedanib also significantly affected corneal neovascularization. Therefore, we scraped off the central $2 \mathrm{~mm}$ of the corneal epithelium before suture placement to promote penetration of the drug into the corneal stroma. By application of $5 \mathrm{mg} / \mathrm{mL}$ nintedanib as eye drops four times daily, the outgrowths of blood $(P<0.01)$ and lymphatic $(P<0.01, \mathrm{n}=8)$ vessels were significantly inhibited compared with the controls ( $\mathrm{n}=8$; Figure 5$)$.

\section{Nintedanib inhibits inflammatory cell recruitment} in the sutured cornea

Finally, we evaluated whether the application of nintedanib suppresses inflammatory cell $\left(\mathrm{F} 4 / 80^{+}\right.$cell) recruitment in the sutured cornea. Notably, a reduction in $\mathrm{F} 4 / 80^{+}$cell infiltration was observed at day 14 in corneas from both systemic $(n=6)$ and topical $(n=6)$ applications of nintedanib. In comparison with controls, recruitment of $\mathrm{F} 4 / 80^{+}$cells was inhibited by $61 \%$ with the systemic application of nintedanib $(P<0.01$; Figure 6C) and by $49 \%$ with the topical application of nintedanib $(P<0.01$; Figure 6D).

\section{Discussion}

The results of our current study allow the following four conclusions to be drawn:

1. Nintedanib, a potent triple angiokinase inhibitor, inhibits both lymphangiogenesis and hemangiogenesis. 


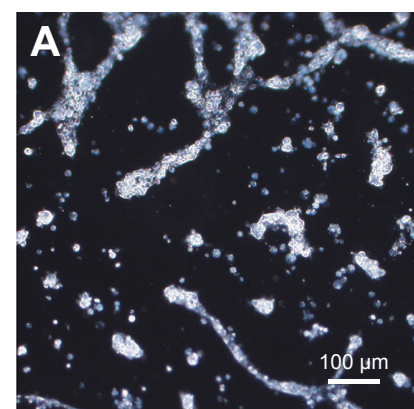

Control

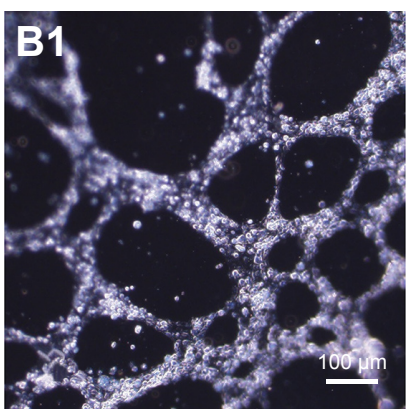

VEGF-C

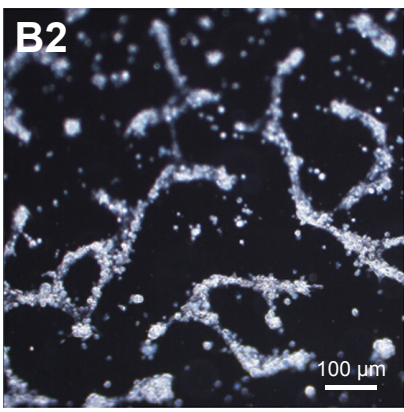

VEGF-C + BIBF 1120

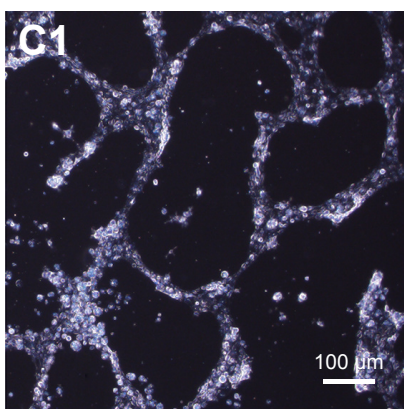

PDGF-BB

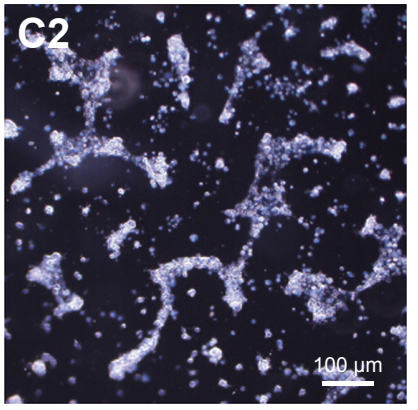

PDGF-BB + BIBF 1120

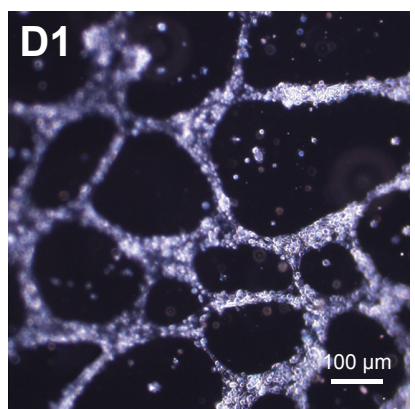

bFGF

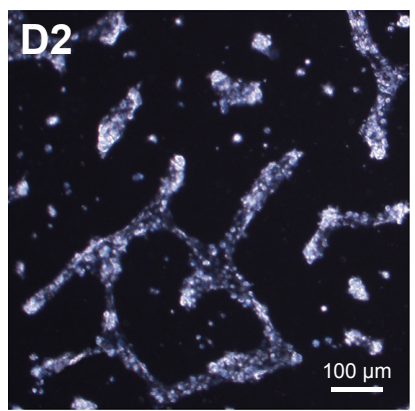

bFGF + BIBF 1120
E

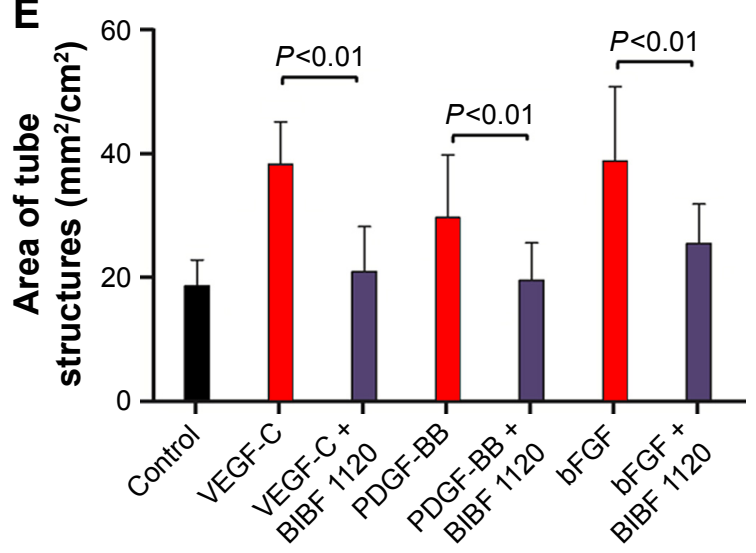

F

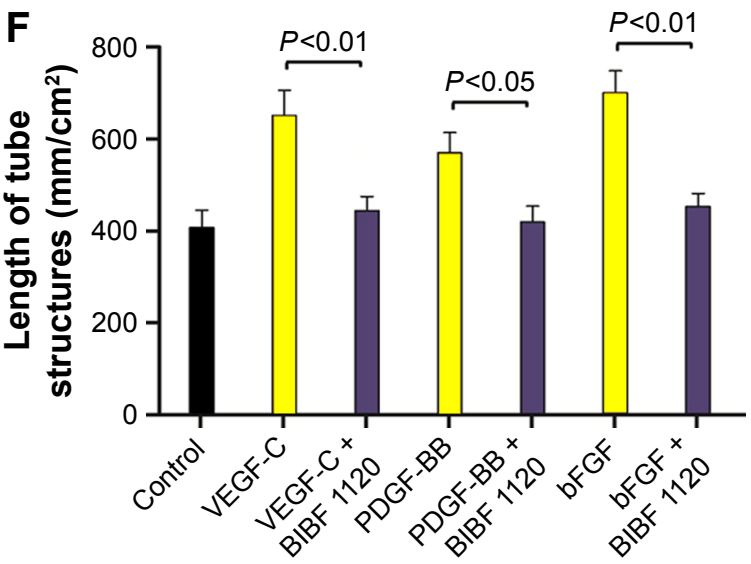

Figure 3 BIBF II 20 (nintedanib) inhibited growth factor-induced tube formation of HLECs.

Notes: (A) Cells treated with $0.1 \%$ DMSO were used as controls. (BI and B2) VEGF-C $(50 \mathrm{ng} / \mathrm{mL})$ enhanced tube formation of HLECs significantly, and the enhanced effect was significantly reduced by $0.1 \mu \mathrm{M}$ nintedanib. (CI and C2) PDGF-BB $(50 \mathrm{ng} / \mathrm{mL})$ enhanced tube formation of HLECs significantly, and the enhanced effect was significantly reduced by $0.1 \mu \mathrm{M}$ nintedanib. (DI and D2) A concentration of $50 \mathrm{ng} / \mathrm{mL}$ of bFGF enhanced tube formation of HLECs significantly, and the enhanced effect was significantly reduced by $0.1 \mu \mathrm{M}$ nintedanib. (E) The area of the tube structure after being stimulated by VEGF-C, PDGF-BB, and bFGF at a concentration of $50 \mathrm{ng} / \mathrm{mL}$ in the presence or absence of $0.1 \mu \mathrm{M}$ nintedanib. (F) The length of the tube structure after being stimulated by VEGF-C, PDGF-BB, and bFGF at a concentration of 50 ng/mL in the presence or absence of $0.1 \mu \mathrm{M}$ nintedanib. A-D at I00X magnification.

Abbreviations: HLECs, human lymphatic endothelial cells; DMSO, dimethyl sulfoxide; VEGF-C, vascular endothelial growth factor-C; PDGF-BB, platelet-derived growth factor-BB; bFGF, basic fibroblast growth factor.

2. Nintedanib prevents enhanced effects of cell proliferation, migration, and tube formation of HLECs stimulated by VEGF-C, PDGF-BB, and bFGF.

3. Both topical and systemic applications of nintedanib inhibit suture-induced hemangiogenesis and lymphangiogenesis in the murine cornea.

4. Nintedanib inhibits inflammatory cell recruitment in the sutured murine cornea.
The first commercially available antiangiogenic agent for cancer treatment was bevacizumab, a humanized monoclonal antibody that blocks VEGF-A. ${ }^{30}$ However, a proposed mechanism of resistance to agents that inhibit VEGF-mediated angiogenesis is tumor cells' ability to escape from sustained VEGF inhibition by upregulating other proangiogenic factors, such as PDGF and FGF. ${ }^{31-33}$ This finding highlights the importance of developing multi-targeted tyrosine kinase 

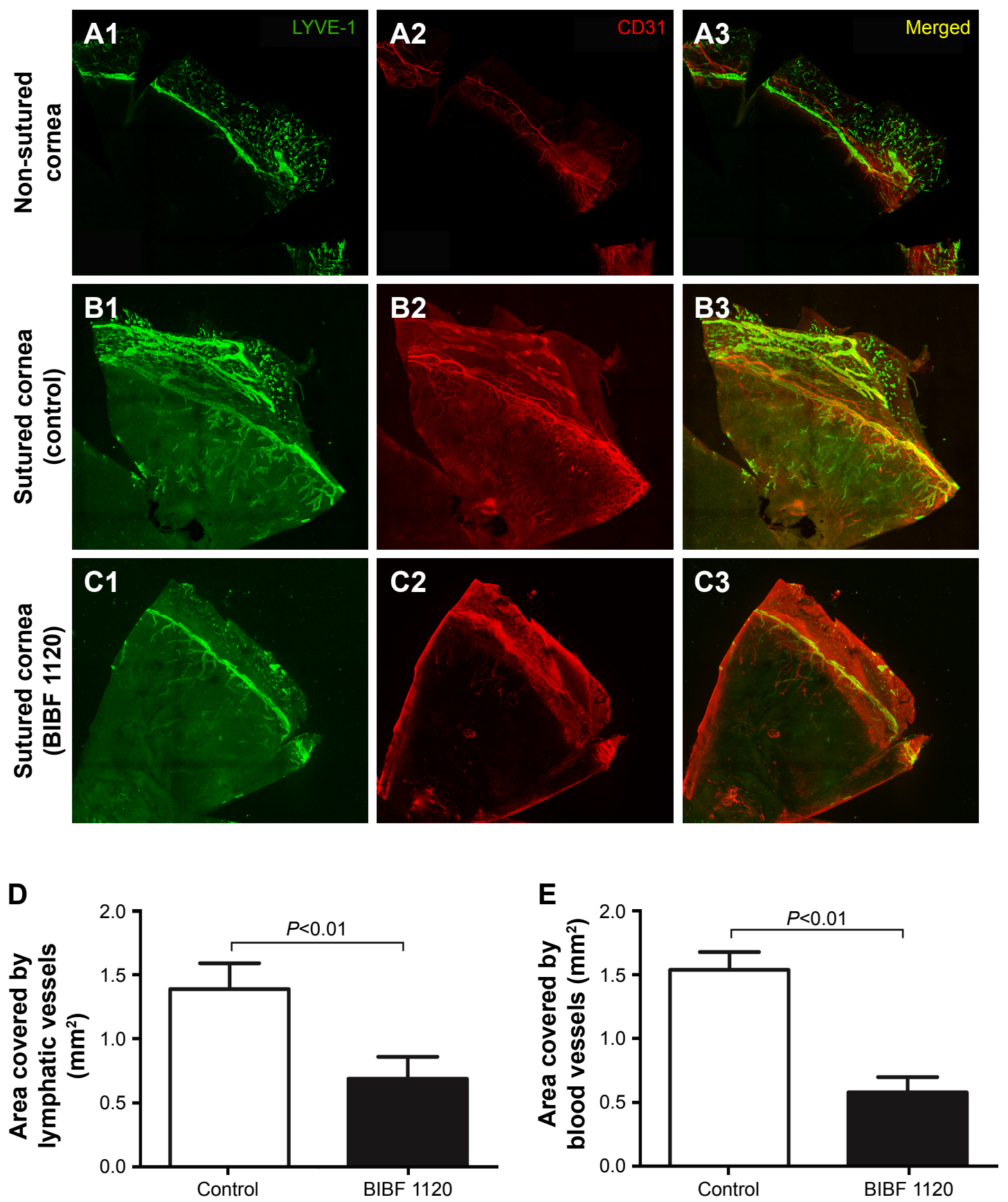

Figure 4 Systemic application of BIBF II 20 (nintedanib) significantly decreased angiogenesis and hemangiogenesis in the cornea in comparison with the controls.

Notes: (AI-A3) Non-sutured corneas were used as negative controls. Sutured corneas with nintedanib treatment $(\mathbf{C l}-\mathbf{C} 3)$ were compared to those without nintedanib treatment (BI-B3). Significant inhibition of $(\mathbf{D})$ lymphangiogenesis $(P<0.0 \mathrm{I}, \mathrm{n}=8)$ and $(\mathbf{E})$ hemangiogenesis $(P<0.0 \mathrm{I}, \mathrm{n}=8)$ after systemic treatment with nintedanib over 14 days was observed compared with the controls without nintedanib treatment. A-C at $100 \times$ magnification.

Abbreviation: LYVE-I, lymphatic vessel endothelial hyaluronan receptor-I.

inhibitors (TKIs) that can block several key receptors and simultaneously maintain the selectivity profile of kinases in terms of safety and tolerability. Novel multi-targeted agents that have antiangiogenic properties, such as sorafenib, sunitinib, vandetanib, and others, have been developed in recent years. ${ }^{34}$ These agents all inhibit additional pathways beyond VEGF signaling. Nintedanib, a triple angiokinase inhibitor of VEGFR, PDGFR, and FGFR, is one of these investigational targeted agents. Nintedanib has been used for treating several malignancies, including non-small cell lung cancer, prostate cancer, and ovarian cancer, because of its potent antiangiogenic ability. ${ }^{35-37}$

Like angiogenesis, lymphangiogenesis has been considered as one of the major components of tumor progression 

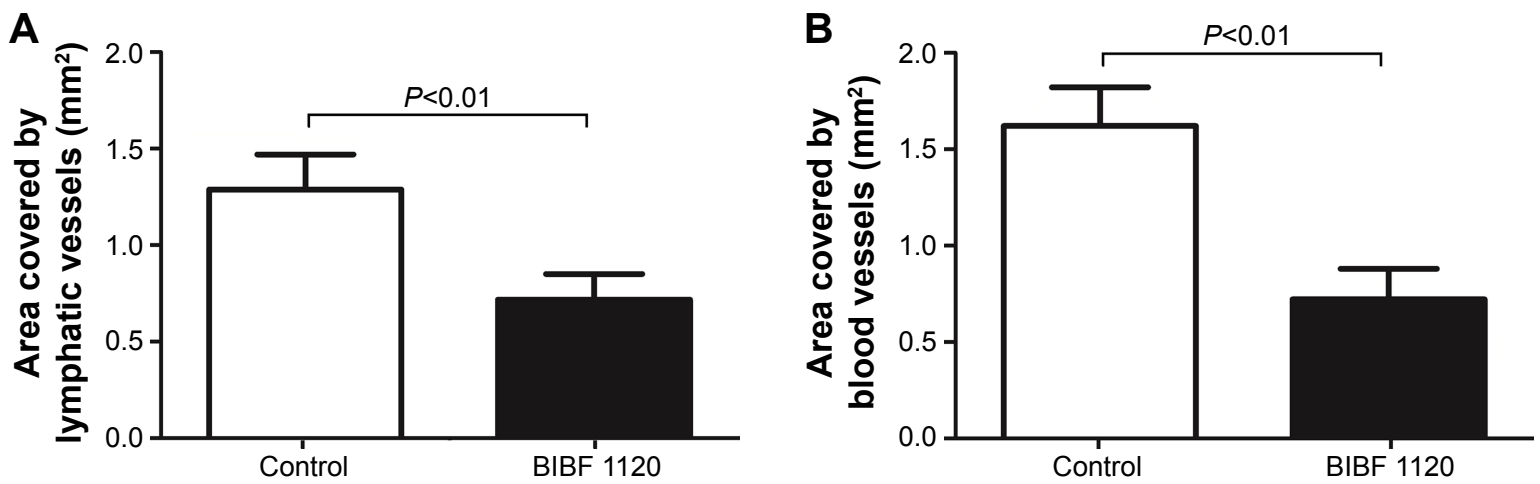

Figure 5 Topical application of BIBF II 20 (nintedanib) significantly decreased lymphangiogenesis and hemangiogenesis in the cornea in comparison with the controls. Note: Significant inhibition of $(\mathbf{A})$ lymphangiogenesis $(P<0.0 \mathrm{I}, \mathrm{n}=8)$ and $(\mathbf{B})$ hemangiogenesis $(P<0.0 \mathrm{I}, \mathrm{n}=8)$ after topical treatment with nintedanib over $\mathrm{I} 2$ days was observed compared with the controls without nintedanib treatment.
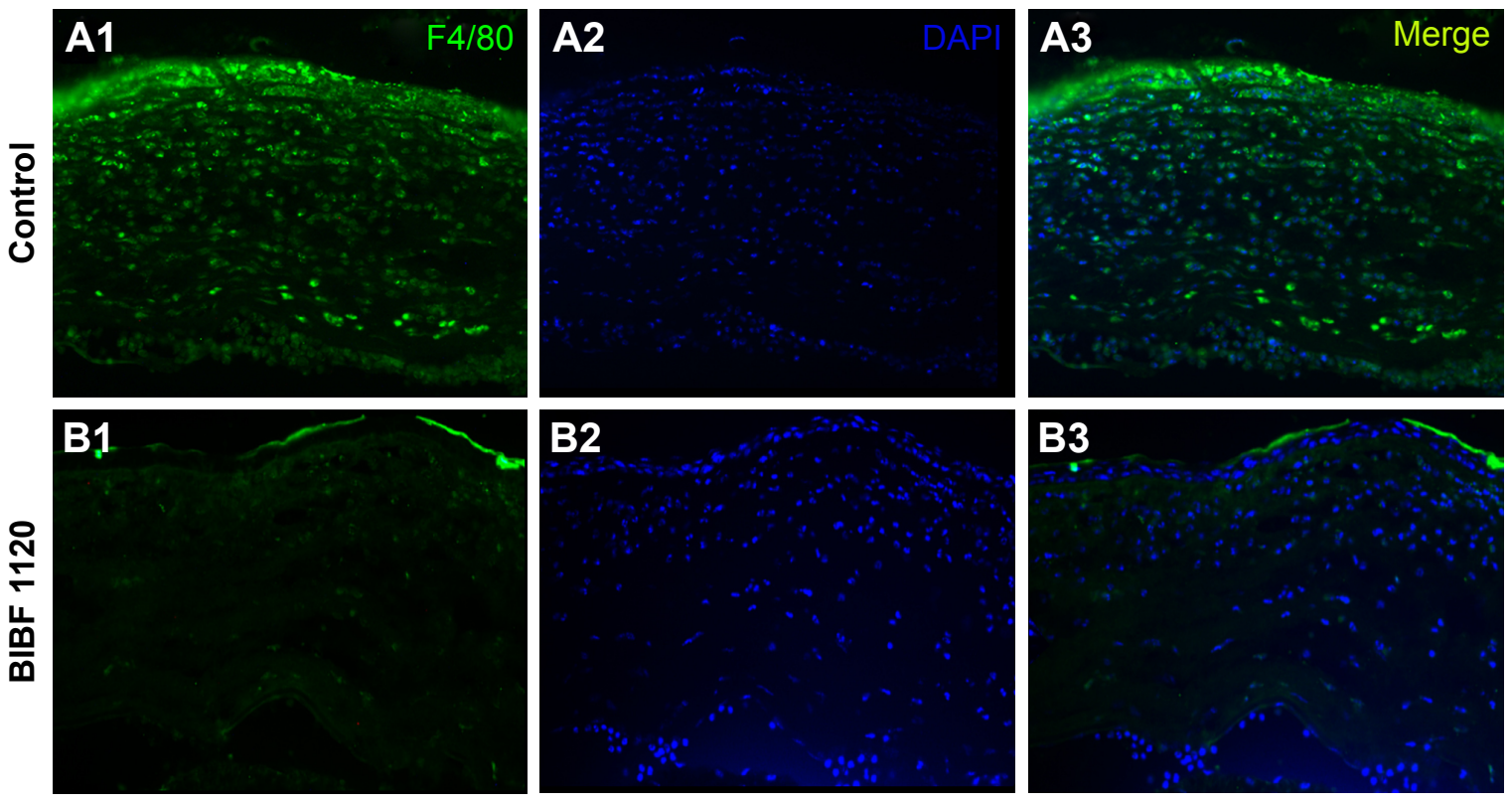

C

Systemic application of BIBF 1120
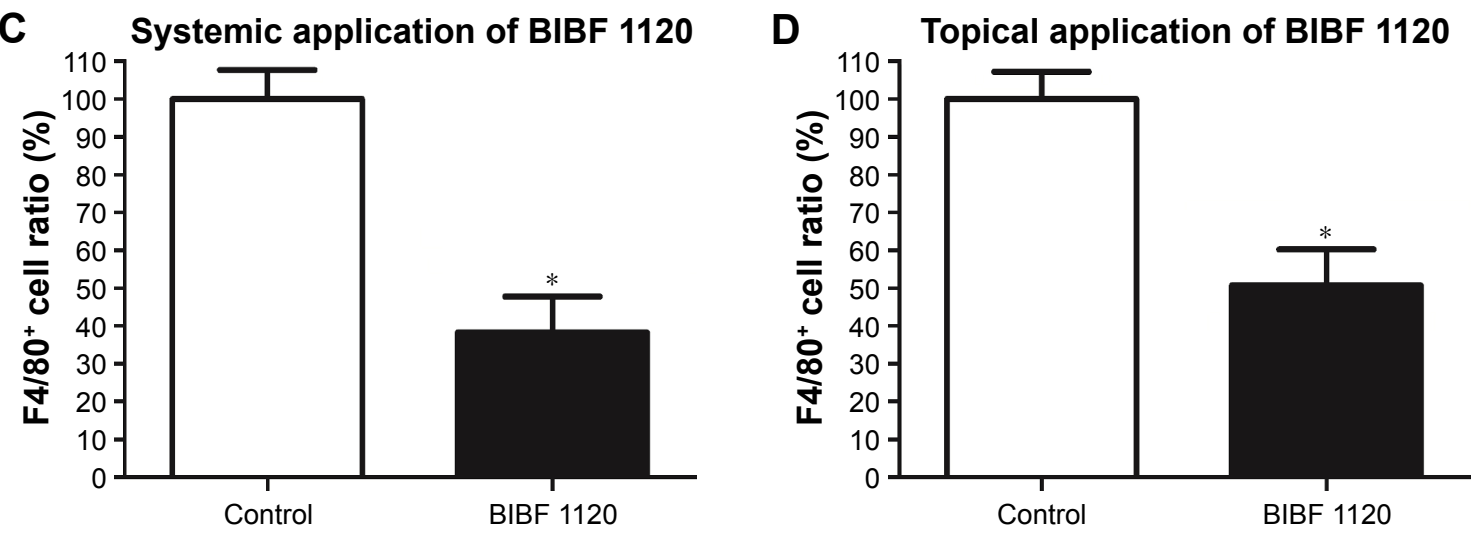

Figure 6 Treatment with BIBF II 20 (nintedanib) significantly diminished the recruitment of inflammatory cells to the cornea.

Notes: (AI-A3) Sutured corneas without systemic nintedanib treatment were infiltrated by numerous F4/80+ cells. (B I-B3) Sutured corneas with systemic nintedanib treatment were infiltrated by a few $\mathrm{F} 4 / 80^{+}$cells. Significant inhibition of inflammatory cell recruitment was observed both with systemic nintedanib treatment and topical nintedanib treatment. In comparison with controls, $61 \%$ of $F 4 / 80^{+}$cell recruitment was inhibited via the systemic application of nintedanib (C), while $49 \%$ was inhibited with the topical application of nintedanib (D). $* P$-value $<0.01$. A-B at $400 \times$ magnification.

Abbreviation: DAPI, 4',6-diamidino-2-phenylindole. 
and metastasis in recent years. ${ }^{38-40}$ Anti-lymphangiogenic treatment has been considered as an effective way to reduce tumor metastasis. Yet, so far, little research has been conducted to investigate the effect of nintedanib on lymphangiogenesis. Thus, our current study provided the evidence of the anti-lymphangiogenic property of nintedanib. In terms of in vitro lymphangiogenesis assay, we evaluated the effect of nintedanib on the biological behavior of growth factor-stimulated HLECs, which included cell proliferation, migration, and tube formation.

Cao et $\mathrm{al}^{41}$ previously showed that VEGF-C and bFGF independently stimulate LEC proliferation and migration; they also found that VEGF-C and bFGF collaboratively stimulated lymphangiogenesis, which occurred under pathological conditions, such as in the tumor environment and corneal neovascularization. Meanwhile, it has been reported that $\mathrm{PDGF}-\mathrm{BB}$ could independently stimulate LEC proliferation and migration and stimulate both tumor and corneal lymphangiogenesis. ${ }^{8,12}$ In the current study, we found that VEGF-C, PDGF-BB, and bFGF could all stimulate LEC proliferation and migration independently, which was similar to the results of previous studies. ${ }^{12,41}$ Furthermore, we observed that these three growth factors could enhance the tube formation of HLECs in vitro. Nintedanib's targets include PDGFR, FGFR, and VEGFR, and its activity results in the blockade of signaling of VEGF/ VEGFR, FGF/FGFR, and PDGF/PDGFR. ${ }^{18}$ Our in vitro experiment showed that this triple angiokinase inhibitor could eliminate the proliferation, migration, and tube formation of HLECs enhanced by VEGF-C, PDGF-BB, and bFGF, thereby indicating its ability to inhibit growth factor-induced lymphangiogenesis.

Following the in vitro assay, we also investigated the in vivo effect of nintedanib on the outgrowth of blood and lymphatic vessels in a suture-induced corneal neovascularization assay. Considering that the cornea is one of the few tissues in the body that is normally devoid of both blood and lymphatic vessels, the murine cornea has been extensively exploited as a tool for vascular studies. ${ }^{42}$ More recently, the use of this unique tissue in the new area of lymphangiogenesis has started to generate promising data. ${ }^{43}$ It is noteworthy that both topical and systemic applications of nintedanib inhibited suture-induced hemangiogenesis and lymphangiogenesis in the murine cornea in the current study. Nintedanib's dual blocking effect on hemangiogenesis and lymphangiogenesis provides more evidence to verify its clinical efficacy for several malignancies by inhibiting tumor progression and metastasis.
In addition to tumor metastasis, lymphangiogenesis also has been suggested to play a key role in graft rejection. ${ }^{44,45}$ Immune-mediated graft rejections remain the most common cause of graft failure after organ and tissue transplantation. A great medical need exists for pharmacological strategies to promote graft survival. Moreover, there has been extensive research concerning the role of lymphangiogenesis in corneal graft rejection. ${ }^{46-48}$ The three structural components of the immune system allowing for immune responses against foreign tissue after transplantation are afferent lymphatic vessels (the afferent arm of the immune reflex arc), regional lymph nodes (the central processing unit), and efferent blood vessels (the efferent arm of the immune reflex arc). ${ }^{46}$ Lymphangiogenesis provides a route for antigens and antigen-presenting cells to reach the regional lymph nodes; thus, it constitutes one of the earliest events in the immune cascade leading to rejection. Anti-lymphangiogenic treatment could be a selective strategy to prevent graft rejection. Thus, it has been suggested that nintedanib has the potential to improve graft survival after penetrating keratoplasty by reducing the ingrowths of both blood and lymphatic vessels, thereby interrupting the so-called immune reflex arc. Since the topical application of nintedanib was proved to be effective for inhibiting corneal hemangiogenesis and lymphangiogenesis in our current study, this suggested a promising topical administration of nintedanib in clinical conditions of ophthalmology. The topical administration is the most common drug delivery and allows easier usage of nintedanib in ophthalmology.

We also provided evidence that, in addition to reducing blood and lymphatic vessel growth, nintedanib was able to inhibit $\mathrm{F} 4 / 80^{+}$cell recruitment. $\mathrm{F} 4 / 80^{+}$cells include macrophages and dendritic cells. Accordingly, other TKIs, such as PTK787/ZK222584, ZK261991, and sunitinib, have been reported to display similar inhibition of inflammatory cell recruitment in a corneal neovascularization model. ${ }^{49,50}$ There were several possible explanations for this finding. First, reduced $\mathrm{F} 4 / 80^{+}$cell infiltration might result from the inhibition of VEGF-A/VEGFR-1 pathway by nintedanib. Infiltrating macrophages represent a major source of prolymph/angiogenic factors, such as VEGF-A, VEGF-C, and VEGF-D, leading to an immune amplification. ${ }^{5}$ Nintedanib might also abrogate the effects of these prolymph/angiogenic factors secreted by macrophages in addition to its impact on inflammatory cell recruitment. Besides, nintedanib also potently inhibits Lck, a downstream target of the T-cell receptor, ${ }^{18}$ which 
may reduce the chemokine for macrophage recruitment produced by effective T-cells.

It has been reported that macrophages can also take part in the formation of lymphatic vessels by transdifferentiating into an endothelial phenotype and incorporating into the lymphatic wall. ${ }^{51,52}$ This evidence suggests an indirect effect of nintedanib on lymphangiogenesis by reducing $\mathrm{F} 4 / 80^{+}$cell recruitment.

Numerous studies have suggested that T-cells play an important role in corneal graft rejection, ${ }^{53-55}$ and the Lck inhibitors have proved to be able to prevent solid organ transplant rejection, including kidney and heart transplantation..$^{56-58}$ This evidence indicated that nintedanib, with the ability of inhibiting Lck, might have an effect to prevent corneal graft rejection by immune suppression. We also verified nintedanib's anti-lymphangiogenic effect in the current study. Therefore, nintedanib, with both properties of anti-lymphangiogenesis and immune suppression, may be more effective in maintaining corneal allograft survival. However, further study should be conducted to confirm this hypothesis in the future.

The previous studies have also demonstrated that corneal fibroblasts play an important role in corneal neovascularization. It was proved that corneal fibroblasts exhibited enhanced synthesis and secretion of a wide variety of proangiogenic factors, including extracellular matrix-degrading enzymes (matrix metalloproteinases 2, 3, and 14) and VEGF in the mouse model of corneal alkali burn. ${ }^{59}$ It was also reported that enhanced VEGF-A expression by corneal fibroblasts was dependent on the bFGF-induced pathway. ${ }^{60}$ Nintedanib has been proved to inhibit fibroblast activation, ${ }^{61,62}$ and this could be another possible reason for the anti-corneal neovascularization by nintedanib.

There were some limitations to this study. We did not investigate the safety of topical application of nintedanib in an in vivo experiment. Since the safety evaluation is quite necessary for the clinical application of a drug, we are planning a further study in order to determine the optimal dosage and safety of topical application of nintedanib for anti-corneal neovascularization. Furthermore, the lymphangiogenesis is generally accompanied by angiogenesis in numerous pathological conditions, such as infection, inflammation, chemical trauma, and tumor, ${ }^{63}$ and these two processes share some common proangiogenic factors such as VEGF, FGF, and PDGF, ${ }^{43}$ which made it difficult to research on lymphangiogenesis completely independent of angiogenesis. Thus, our current study failed to differentiate the specific mechanism of nintedanib's anti-lymphangiogenic functions versus its anti-angiogenesis functions.

\section{Conclusion}

In summary, nintedanib, a novel potent triple angiokinase inhibitor, was shown to inhibit lymphangiogenesis both in vitro and in vivo, in addition to its antiangiogenic property. Nintedanib inhibits lymphangiogenesis in vitro stimulated by growth factors, including VEGF-C, bFGF, and PDGF-BB. Applied topically or systemically, it effectively alleviates suture-induced corneal hemangiogenesis and lymphangiogenesis accompanied by reducing inflammatory cell recruitment, thereby representing a new promising treatment for graft rejection after penetrating keratoplasty.

\section{Acknowledgment}

We gratefully acknowledge Gang Li and Rong Zhang from the Department of Ophthalmology, Eye and ENT Hospital, Fudan University, and Key Laboratory of Myopia, Ministry of Health for their excellent technical assistance.

\section{Disclosure}

The authors report no competing interests or conflicts of interest in this work.

\section{References}

1. Detry B, Bruyere F, Erpicum C, et al. Digging deeper into lymphatic vessel formation in vitro and in vivo. BMC Cell Biol. 2011;12:29.

2. Jussila L, Alitalo K. Vascular growth factors and lymphangiogenesis. Physiol Rev. 2002;82(3):673-700.

3. Avraamides CJ, Garmy-Susini B, Varner JA. Integrins in angiogenesis and lymphangiogenesis. Nat Rev Cancer. 2008;8(8):604-617.

4. Paupert J, Sounni NE, Noël A. Lymphangiogenesis in post-natal tissue remodeling: lymphatic endothelial cell connection with its environment. Mol Aspects Med. 2011;32(2):146-158.

5. Cursiefen C, Chen L, Borges LP, et al. VEGF-A stimulates lymphangiogenesis and hemangiogenesis in inflammatory neovascularization via macrophage recruitment. J Clin Invest. 2004;113(7):1040-1050.

6. Hajrasouliha AR, Funaki T, Sadrai Z, Hattori T, Chauhan SK, Dana R. Vascular endothelial growth factor-C promotes alloimmunity by amplifying antigen-presenting cell maturation and lymphangiogenesis. Invest Ophthalmol Vis Sci. 2012;53(3):1244-1250.

7. Chang LK, Garcia-Cardeña G, Farnebo F, et al. Dose-dependent response of FGF-2 for lymphangiogenesis. Proc Natl Acad Sci U S A. 2004; 101(32):11658-11663.

8. Cao Y. Direct role of PDGF-BB in lymphangiogenesis and lymphatic metastasis. Cell Cycle. 2005;4(2):228-230.

9. Karkkainen MJ, Haiko P, Sainio K, et al. Vascular endothelial growth factor $\mathrm{C}$ is required for sprouting of the first lymphatic vessels from embryonic veins. Nat Immunol. 2004;5(1):74-80.

10. Al-Rawi MAA, Jiang WG. Lymphangiogenesis and cancer metastasis. Front Biosci. 2011;16:723-739.

11. Tzeng HE, Chang AC, Tsai CH, Wang SW, Tang CH. Basic fibroblast growth factor promotes VEGF-C-dependent lymphangiogenesis via inhibition of miR-381 in human chondrosarcoma cells. Oncotarget. 2016;7(25):38566-38578.

12. Cao R, Björndahl MA, Religa P, et al. PDGF-BB induces intratumoral lymphangiogenesis and promotes lymphatic metastasis. Cancer Cell. 2004;6(4):333-345. 
13. Cao Y, Zhong W. Tumor-derived lymphangiogenic factors and lymphatic metastasis. Biomed Pharmacother. 2007;61(9):534-539.

14. Van der Auwera I, Cao Y, Tille JC, et al. First international consensus on the methodology of lymphangiogenesis quantification in solid human tumours. Br J Cancer. 2006;95(12):1611-1625.

15. Chen L. Ocular lymphatics: state-of-the-art review. Lymphology. 2009;42(2):66-76.

16. Alitalo K, Tammela T, Petrova TV. Lymphangiogenesis in development and human disease. Nature. 2005;438(7070):946-953.

17. Cueni LN, Detmar M. The lymphatic system in health and disease. Lymphat Res Biol. 2008;6(3-4):109-122.

18. Hilberg F, Roth GJ, Krssak M, et al. BIBF 1120: triple angiokinase inhibitor with sustained receptor blockade and good antitumor efficacy. Cancer Res. 2008;68(12):4774-4782.

19. Kutluk Cenik B, Ostapoff KT, Gerber DE, Brekken RA. BIBF 1120 (nintedanib), a triple angiokinase inhibitor, induces hypoxia but not EMT and blocks progression of preclinical models of lung and pancreatic cancer. Mol Cancer Ther. 2013;12(6):992-1001.

20. Mross K, Stefanic M, Gmehling D, et al. Phase I study of the angiogenesis inhibitor BIBF 1120 in patients with advanced solid tumors. Clin Cancer Res. 2010;16(1):311-319.

21. Reck M, Kaiser R, Eschbach C, et al. A phase II double-blind study to investigate efficacy and safety of two doses of the triple angiokinase inhibitor BIBF 1120 in patients with relapsed advanced non-small-cell lung cancer. Ann Oncol. 2011;22(6):1374-1381.

22. Ledermann JA, Hackshaw A, Kaye S, et al. Randomized phase II placebo-controlled trial of maintenance therapy using the oral triple angiokinase inhibitor BIBF 1120 after chemotherapy for relapsed ovarian cancer. J Clin Oncol. 2011;29(28):3798-3804.

23. Rivera JC, Noueihed B, Omri S, Barrueco J, Hilberg F, Chemtob S BIBF1120 (Vargatef) inhibits preretinal neovascularization and enhances normal vascularization in a model of vasoproliferative retinopathy. Invest Ophthalmol Vis Sci. 2015;56(13):7897-7907.

24. Aguilar B, Choi I, Choi D, et al. Lymphatic reprogramming by Kaposi sarcoma herpes virus promotes the oncogenic activity of the virus-encoded G-protein-coupled receptor. Cancer Res. 2012;72(22):5833-5842.

25. Ding D, Wei S, Song Y, et al. Osthole exhibits anti-cancer property in rat glioma cells through inhibiting PI3K/Akt and MAPK signaling pathways. Cell Physiol Biochem. 2013;32(6):1751-1760.

26. Min JK, Cho YL, Choi JH, et al. Receptor activator of nuclear factor (NF)-kappaB ligand (RANKL) increases vascular permeability: impaired permeability and angiogenesis in eNOS-deficient mice. Blood. 2007;109(4):1495-1502.

27. Truong T, Huang E, Yuen D, Chen L. Corneal lymphatic valve formation in relation to lymphangiogenesis. Invest Ophthalmol Vis Sci. 2014;55(3): 1876-1883.

28. Anderson C, Zhou Q, Wang S. An alkali-burn injury model of corneal neovascularization in the mouse. J Vis Exp. 2014;(86):51159.

29. Kojima T, Chang JH, Azar DT. Proangiogenic role of ephrinB1/ EphB1 in basic fibroblast growth factor-induced corneal angiogenesis. Am J Pathol. 2007;170(2):764-773.

30. Ferrara N, Hillan KJ, Gerber HP, Novotny W. Discovery and development of bevacizumab, an anti-VEGF antibody for treating cancer. Nat Rev Drug Discov. 2004;3(5):391-400.

31. Erber R, Thurnher A, Katsen AD, et al. Combined inhibition of VEGF and PDGF signaling enforces tumor vessel regression by interfering with pericyte-mediated endothelial cell survival mechanisms. FASEB J. 2004;18(2):338-340

32. Bergers G, Song S, Meyer-Morse N, Bergsland E, Hanahan D. Benefits of targeting both pericytes and endothelial cells in the tumor vasculature with kinase inhibitors. J Clin Invest. 2003;111(9):1287-1295.

33. Casanovas O, Hicklin DJ, Bergers G, Hanahan D. Drug resistance by evasion of antiangiogenic targeting of VEGF signaling in late-stage pancreatic islet tumors. Cancer Cell. 2005;8(4):299-309.

34. Santos ES, Gomez JE, Raez LE. Targeting angiogenesis from multiple pathways simultaneously: BIBF 1120, an investigational novel triple angiokinase inhibitor. Invest New Drugs. 2012;30(3):1261-1269.
35. Caglevic C, Grassi M, Raez L, et al. Nintedanib in non-small cell lung cancer: from preclinical to approval. Ther Adv Respir Dis. 2015;9(4): $164-172$.

36. Roth GJ, Binder R, Colbatzky F, et al. Nintedanib: from discovery to the clinic. J Med Chem. 2015;58(3):1053-1063.

37. du Bois A, Kristensen G, Ray-Coquard I, et al; AGO Study Group led Gynecologic Cancer Intergroup/European Network of Gynaecologic Oncology Trials Groups Intergroup Consortium. Standard first-line chemotherapy with or without nintedanib for advanced ovarian cancer (AGO-OVAR 12): a randomised, double-blind, placebo-controlled phase 3 trial. Lancet Oncol. 2016;17(1):78-89.

38. Garmy-Susini B, Avraamides CJ, Schmid MC, et al. Integrin alpha4beta1 signaling is required for lymphangiogenesis and tumor metastasis. Cancer Res. 2010;70(8):3042-3051.

39. Cao Z, Shang B, Zhang G, et al. Tumor cell-mediated neovascularization and lymphangiogenesis contrive tumor progression and cancer metastasis. Biochim Biophys Acta. 2013;1836(2):273-286.

40. Hirakawa S, Brown LF, Kodama S, Paavonen K, Alitalo K, Detmar M. VEGF-C-induced lymphangiogenesis in sentinel lymph nodes promotes tumor metastasis to distant sites. Blood. 2007;109(3):1010-1017.

41. Cao R, Ji H, Feng N, et al. Collaborative interplay between FGF-2 and VEGF-C promotes lymphangiogenesis and metastasis. Proc Natl Acad Sci U S A. 2012;109(39):15894-15899.

42. Kather JN, Kroll J. Transgenic mouse models of corneal neovascularization: new perspectives for angiogenesis research. Invest Ophthalmol Vis Sci. 2014;55(11):7637-7651.

43. Ellenberg D, Azar DT, Hallak JA, et al. Novel aspects of corneal angiogenic and lymphangiogenic privilege. Prog Retin Eye Res. 2010; 29(3):208-248.

44. Kerjaschki D, Huttary N, Raab I, et al. Lymphatic endothelial progenitor cells contribute to de novo lymphangiogenesis in human renal transplants. Nat Med. 2006;12(2):230-234.

45. Yamamoto I, Yamaguchi Y, Yamamoto H, et al. A pathological analysis of lymphatic vessels in early renal allograft. Transplant Proc. 2006;38(10):3300-3303.

46. Dietrich T, Bock F, Yuen D, et al. Cutting edge: lymphatic vessels, not blood vessels, primarily mediate immune rejections after transplantation. J Immunol. 2010;184(2):535-539.

47. Tang XL, Sun JF, Wang XY, Du LL, Liu P. Blocking neuropilin-2 enhances corneal allograft survival by selectively inhibiting lymphangiogenesis on vascularized beds. Mol Vis. 2010;16:2354-2361.

48. Zhang H, Grimaldo S, Yuen D, Chen L. Combined blockade of VEGFR-3 and VLA-1 markedly promotes high-risk corneal transplant survival. Invest Ophthalmol Vis Sci. 2011;52(9):6529-6535.

49. Hos D, Bock F, Dietrich T, et al. Inflammatory corneal (lymph)angiogenesis is blocked by VEGFR-tyrosine kinase inhibitor ZK 261991, resulting in improved graft survival after corneal transplantation. Invest Ophthalmol Vis Sci. 2008;49(5):1836-1842.

50. Detry B, Blacher S, Erpicum C, et al. Sunitinib inhibits inflammatory corneal lymphangiogenesis. Invest Ophthalmol Vis Sci. 2013;54(5): 3082-3093.

51. Gordon EJ, Rao S, Pollard JW, Nutt SL, Lang RA, Harvey NL. Macrophages define dermal lymphatic vessel calibre during development by regulating lymphatic endothelial cell proliferation. Development. 2010;137(22):3899-3910.

52. Maruyama K, Ii M, Cursiefen C, et al. Inflammation-induced lymphangiogenesis in the cornea arises from CD11b-positive macrophages. J Clin Invest. 2005;115(9):2363-2372.

53. Nicholls SM, Mitchard LK, Laycock GM, et al. A model of corneal graft rejection in semi-inbred NIH miniature swine: significant T-cell infiltration of clinically accepted allografts. Invest Ophthalmol Vis Sci. 2012;53(6):3183-3192.

54. Tan Y, Cruz-Guilloty F, Medina-Mendez CA, et al. Immunological disruption of antiangiogenic signals by recruited allospecific $\mathrm{T}$ cells leads to corneal allograft rejection. J Immunol. 2012;188(12):5962-5969.

55. Tan Y, Abdulreda MH, Cruz-Guilloty F, et al. Role of T cell recruitment and chemokine-regulated intra-graft $\mathrm{T}$ cell motility patterns in corneal allograft rejection. Am J Transplant. 2013;13(6):1461-1473. 
56. Borhani DW, Calderwood DJ, Friedman MM, et al. A-420983: a potent, orally active inhibitor of lck with efficacy in a model of transplant rejection. Bioorg Med Chem Lett. 2004;14(10):2613-2616.

57. Stachlewitz RF, Hart MA, Bettencourt B, et al. A-770041, a novel and selective small-molecule inhibitor of Lck, prevents heart allograft rejection. J Pharmacol Exp Ther. 2005;315(1):36-41.

58. Burchat A, Borhani DW, Calderwood DJ, Hirst GC, Li B, Stachlewitz RF. Discovery of A-770041, a src-family selective orally active lck inhibitor that prevents organ allograft rejection. Bioorg Med Chem Lett. 2006;16(1):118-122.

59. Zhou Q, Yang L, Qu M, et al. Role of senescent fibroblasts on alkaliinduced corneal neovascularization. J Cell Physiol. 2012;227(3): $1148-1156$.
60. Han KY, Fahd DC, Tshionyi M, et al. MT1-MMP modulates bFGFinduced VEGF-A expression in corneal fibroblasts. Protein Pept Lett. 2012;19(12):1334-1339.

61. Hostettler KE, Zhong J, Papakonstantinou E, et al. Anti-fibrotic effects of nintedanib in lung fibroblasts derived from patients with idiopathic pulmonary fibrosis. Respir Res. 2014;15:157.

62. Huang J, Beyer C, Palumbo-Zerr K, et al. Nintedanib inhibits fibroblast activation and ameliorates fibrosis in preclinical models of systemic sclerosis. Ann Rheum Dis. 2016;75(5):883-890.

63. Karpanen T, Alitalo K. Molecular biology and pathology of lymphangiogenesis. Annu Rev Pathol. 2008;3:367-397.

\section{Publish your work in this journal}

Drug Design, Development and Therapy is an international, peerreviewed open-access journal that spans the spectrum of drug design and development through to clinical applications. Clinical outcomes, patient safety, and programs for the development and effective, safe, and sustained use of medicines are the features of the journal, which has also been accepted for indexing on PubMed Central. The manuscript management system is completely online and includes a very quick and fair peer-review system, which is all easy to use. Visit http://www.dovepress.com/testimonials.php to read real quotes from published authors.

Submit your manuscript here: http://www.dovepress.com/drug-design-development-and-therapy-journal 